\title{
Detección de Movimiento de Vehículos en Secuencias de Video Basados en la Diferencia Absoluta entre Fotogramas y la Combinación de Bordes
}

\author{
José Portillo-Portillo, Gabriel Sánchez-Pérez, Jesús Olivares-Mercado, Héctor Pérez-Meana \\ Instituto Politécnico Nacional, Sección de Estudios de Posgrado e Investigación, Escuela Superior de \\ Ingeniería Mecánica y Eléctrica Unidad Culhuacán, Av. Santa Ana No. 1000, Col. San Francisco Culhuacán, \\ 04430, México D.F.-México (e-mail: portillo22@msn.com, caaann@gmail.com, \\ solid_snake98@hotmail.com, hmperezm@ipn.mx)
}

Recibido Dic. 4, 2013; Aceptado Feb. 5, 2014; Versión final recibida Mar. 19, 2014

\begin{abstract}
Resumen
Se presenta un método para la detección de movimiento en secuencias de video, mediante la combinación de la Diferenciación Absoluta entre dos Fotogramas (DAF) y el análisis de bordes en la región considerada movimiento en el tiempo t. Esto permite resolver el fenómeno de apertura del primer plano al unir las regiones que pertenecen a un objeto en movimiento, una de las principales desventajas de la DAF. Las imágenes que son consideradas objetos de interés se someten a operaciones morfológicas para eliminar objetos con componentes conectados menores a un umbral dado. Se realiza una segunda diferenciación absoluta de fotogramas considerando solo las regiones determinadas con movimiento en la primera diferenciación, de manera que al combinar los bordes con la detección de movimiento de la segunda diferencia absoluta, se obtiene la imagen del primer plano. El algoritmo desarrollado tiene la ventaja de presentar la forma y principales parámetros del objeto en movimiento.
\end{abstract}

Palabras clave: fotogramas, apertura en primer plano, detección de movimiento, detección de bordes

\section{Movement Detection of Vehicles in Video Sequences Based on the Absolute Difference Between Frames and Edge Combination}

\begin{abstract}
A method for motion detection in video sequences, through the combination of absolute differentiation between two frames (ADF) and the analysis of edges in the motion region in the frame at time $t$ is presented. This allows solving some disadvantages of the ADF by joining regions that belong to a single object in motion. The images, that are considered to be objects of interest, undergo morphological operations to remove objects with connected components that fall below a given threshold. A second absolute differentiation of frames is carried out considering only specific regions with the first differentiation motion, so that after combining the edges with the motion detection of the second absolute difference, the foreground image is obtained. The algorithm developed has the advantage of depicting the shape and the main parameters that define the object in motion.
\end{abstract}

Keywords: frames, aperture problem, motion detection, edge detection 


\section{INTRODUCCIÓN}

Diversas aplicaciones requieren tener conocimiento acerca del flujo vehicular, lo cual requiere la extracción de las características de los vehículos en movimiento, ya que esto permite usar sistemas complejos como el ITS (sistemas de transporte inteligente, por sus siglas en ingles). Mediante este sistema se pretende un manejo más eficiente de la infraestructura vial, o el desarrollo de nuevas vialidades con la información ITS. Para implementar estos sistemas se requiere conocer parámetros como niveles de ocupación vehicular de los carriles, velocidad vehicular promedio de los carriles, la cantidad de vehículos que transitan por carril, entre otros, así como el uso de sensores económicos y no invasivos como las video cámaras, que permitan afrontar de forma rápida y económica el análisis del flujo vehicular (Padmadas et al., 2010). Estos parámetros pueden extraerse de las secuencias de video, usando detección de movimiento, para lo cual se considerará el movimiento rígido, es decir el movimiento de vehículos. En general, la detección de objetos en movimiento se enfoca en determinar el primer plano (los objetos en movimiento) y el segundo plano (la escena que no cambia o el movimiento que no es de interés). Los métodos comúnmente usados para este propósito pueden resumirse en (Wang et al., 2008): métodos de diferenciación entre fotogramas, métodos de actualización del segundo plano y métodos basado en el flujo óptico, etc. La detección de movimiento mediante la diferenciación entre fotogramas, se realiza usualmente entre los niveles de grises de dos o tres fotogramas adyacentes, de donde se obtiene la región de movimiento usando umbrales. El método usando la actualización del segundo plano consiste en una diferenciación entre el fotograma actual y un fotograma denominado de segundo plano, o de referencia, donde nuevamente para la determinación del primer plano se usa un umbral (Xiaofeng et al.,2010). En este método es importante actualizar el fotograma de referencia de manera que el primer plano este compuesto por el movimiento de interés. Método basado en el flujo óptico, refleja la tendencia de los cambios aparentes en los patrones de intensidad para cada pixel en la escena (Buch et al., 2011).

En los sistemas de detección de movimiento para video vigilancia basados en la diferencia entre dos fotogramas consecutivos (Cao y Li, 2009), las variaciones entre los pixeles correspondientes espacialmente entre dos fotogramas son generadas por el desplazamiento de un objeto considerado de interés, que en adelante denominaremos primer plano, y las variaciones debido a cambios de iluminación, sombras, condiciones climáticas, movimientos de las hojas de los árboles, entre otros, que son considerados como "ruido", que en conjunto con los pixeles que no presentan variación forman el segundo plano. Una de las principales tareas de la detección de movimiento es determinar si una variación en los niveles de intensidad de un pixel se debe al movimiento de un objeto de interés o es debido al ruido, para lo cual en este trabajo se utiliza una diferencia absoluta pixel a pixel, entre dos tramas consecutivas, considerando el espacio de color HSV (Tono, Saturación, Valor) y trabajando sobre el canal de Tono $(\mathrm{H})$. Una de las principales deficiencias del Método basado en la diferencia entre fotogramas, es el fenómeno conocido como "problema de apertura" en el primer plano, y que consiste en que las regiones que se han detectado como movimiento poseen valores homogéneos en los niveles de intensidad; y por tanto el algoritmo de detección de movimiento los considera parte del segundo plano (Utasi y Czúni, 2007).

En este trabajo se propone un algoritmo para la detección de movimiento no articulado en el cual se usa un filtro promedio modificado, para eliminar el ruido que se encuentra separado de los objetos de interés, después de este proceso, se realiza una operación morfológica de dilatación con la finalidad de unir uno o más objetos de interés y calcular los bordes en el tiempo t, y al combinar estos resultados, obtener un solo objeto de aquellos que fueron separados por el fenómeno de apertura. Resultados experimentales muestran que cuando un solo objeto es separado por el fenómeno de apertura, el método propuesto ha sido capaz de unir las partes del objeto, manteniendo el contorno del objeto. El desarrolló y pruebas del método propuesto, se efectúan sobre un video "Propio" denominado "Cisco2500-01" el cual consta de 96882 fotogramas de $240 \times 352$ pixeles, a una taza de 30 fotogramas por segundo, por tanto, cuando hacemos referencia a algún fotograma, se hace referencia a este video.

\section{MÉTODO PROPUESTO}

La Fig. 1, muestra el diagrama a bloques del sistema propuesto en el cual, inicialmente se captura la secuencia de video y se convierte cada fotograma a niveles de gris para su procesamiento. Seguidamente se obtiene una primera aproximación de movimiento mediante la diferencia entre el fotograma actual y el anterior. Seguidamente se procede apurar la estimación del movimiento eliminando el ruido presente en la estimación. Seguidamente se procede a realizar una segunda estimación mediante la diferencia de los fotograma procesados anterior y el actual. Finalmente empleando detección de bordes, junto con operaciones morfológicas se obtiene la detección de movimiento deseada. 


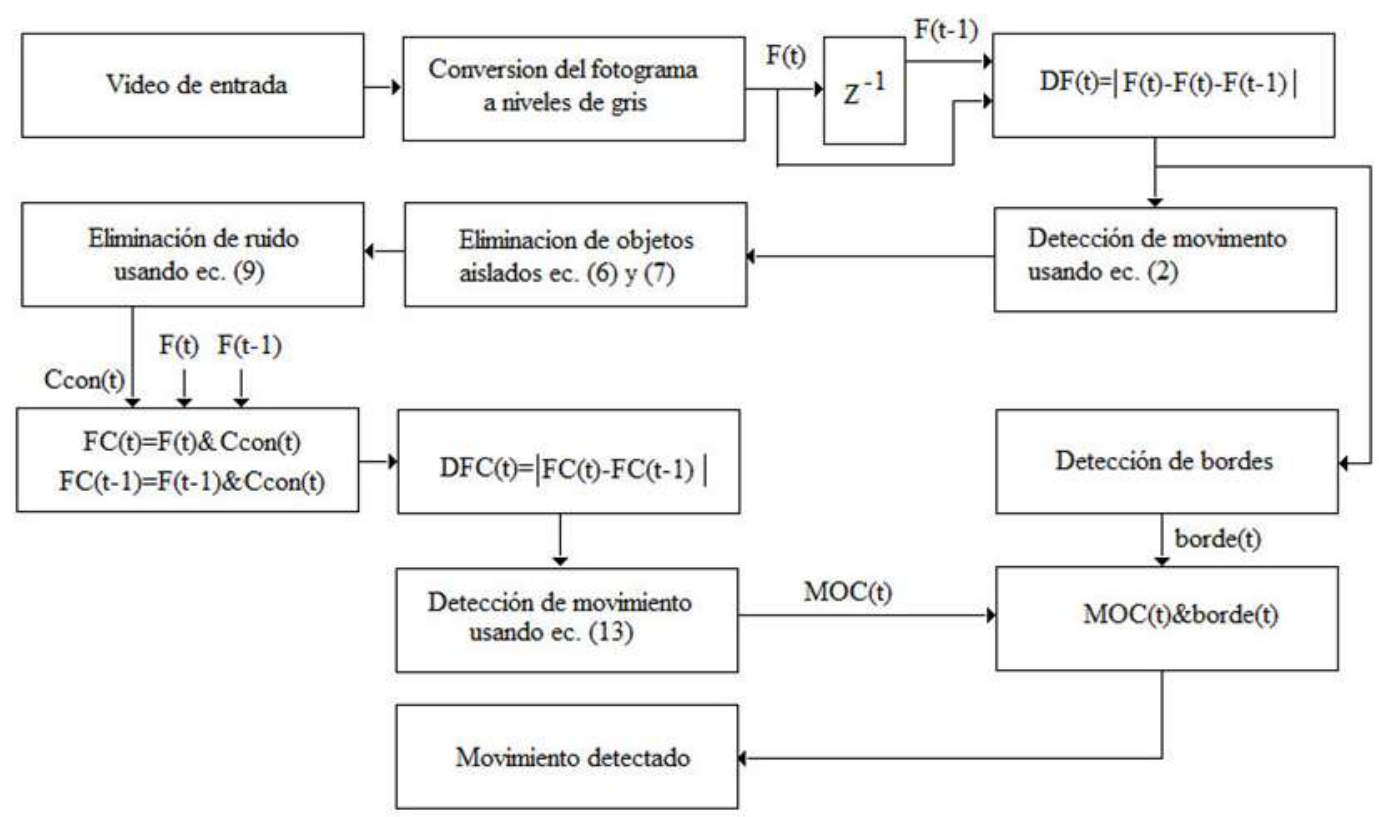

Fig. 1. Sistema propuesto para la detección de movimiento.

Aplicando una diferencia absoluta entre dos fotogramas consecutivos de una secuencia de imágenes en t, se obtiene una aproximación de la región considerada como movimiento,

$D F_{i, j}(t)=\left|F_{i, j}(t)-F_{i, j}(t-1)\right|$

donde $F_{i, j}^{t}$ es el fotograma en el tiempo t. Aquí el valor absoluto de la diferencia se realiza entre el pixel (i,j) y su correspondiente pixel espacial en el tiempo t-1. Para una primera aproximación que determine si la variación de los niveles de intensidad corresponden a un movimiento de interés, se propone la binarización de la imagen mediante un umbral dado por

$M O_{i, j}(t)=\left\{\begin{array}{cc}1, & D F_{i, j}(t) \geq U_{1}(t) \\ 0, & \text { otrocaso }\end{array}\right.$

De aquí consideramos que existe movimiento en el pixel $M O_{i, j}^{t}$ en el tiempo t, cuando $M O_{i, j}^{t}$ es mayor que un umbral cual es determinado como:

$U_{1}^{t}=\bar{x}^{t}+k^{t} \sigma^{t}$

donde $\overline{x^{t}}$ es el valor promedio del valor absoluto de la diferencia entre los fotogramas consecutivos $D F_{i, j}^{t}$, y

${ }^{t}$ es la desviación estándar correspondiente. Estos parámetros estadísticos son calculados para cada momento t, como se muestra en (3) y (4) respectivamente,

$$
\begin{aligned}
& \bar{x}(t)=\frac{1}{m n} \sum_{i=1}^{m} \sum_{j=1}^{n} D F_{i, j}(t) \\
& \sigma(t)=\sqrt{\frac{\sum_{i=1}^{m} \sum_{j=1}^{n}\left(D F_{i, j}(t)-\bar{x}(t)\right)^{2}}{m n}}
\end{aligned}
$$

donde $\mathrm{m}$ y $\mathrm{n}$ son el número de filas y columnas de la imagen que se está procesando. 


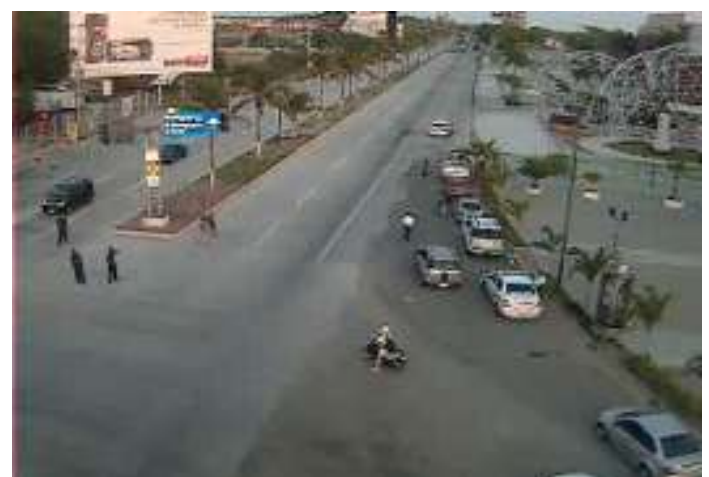

a)

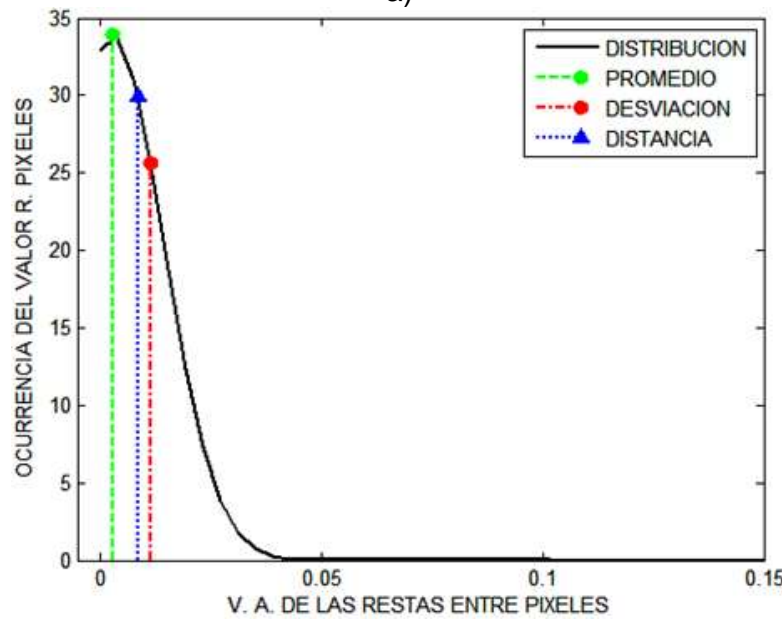

C)

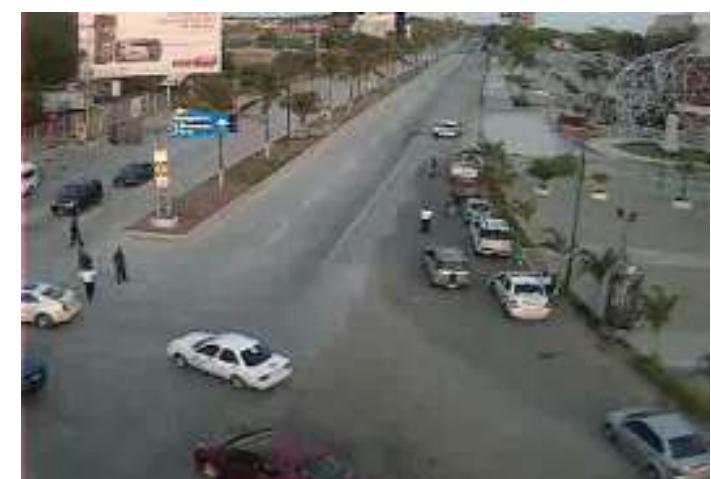

b)

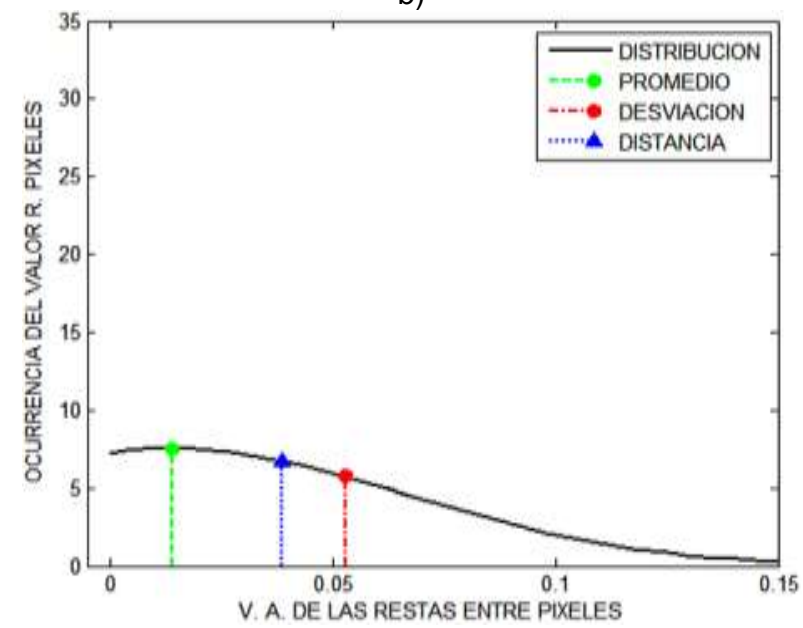

d)

Fig. 2 a) Fotograma 42802, escena sin movimiento; b) Fotograma 42910, escena con movimiento; c) PDF de la d. absoluta entre los Fotograma 42802 y 42801; d) PDF de la d. absoluta entre los Fotograma 42910 y 42911.

Las Figs. 2a) y 2b) muestran los fotogramas 42802 y 42910 que son escenas sin y con movimiento, respectivamente. La gráfica de la función de densidad de probabilidad (PDF) en la Fig. 2c) es bastante estrecha debido a las pocas variaciones que existen entre los fotogramas 42801 y 42802 , en tanto que la gráfica de la PDF en la figura 2d) está más ensanchada debido al desplazamiento de objetos entre los fotogramas 42910 y 42911. Una vez obtenidos el valor promedio, la desviación estándar y la diferencia entre estos a partir de cada fotograma se realiza una gráfica como la mostrada en la Fig. 3.

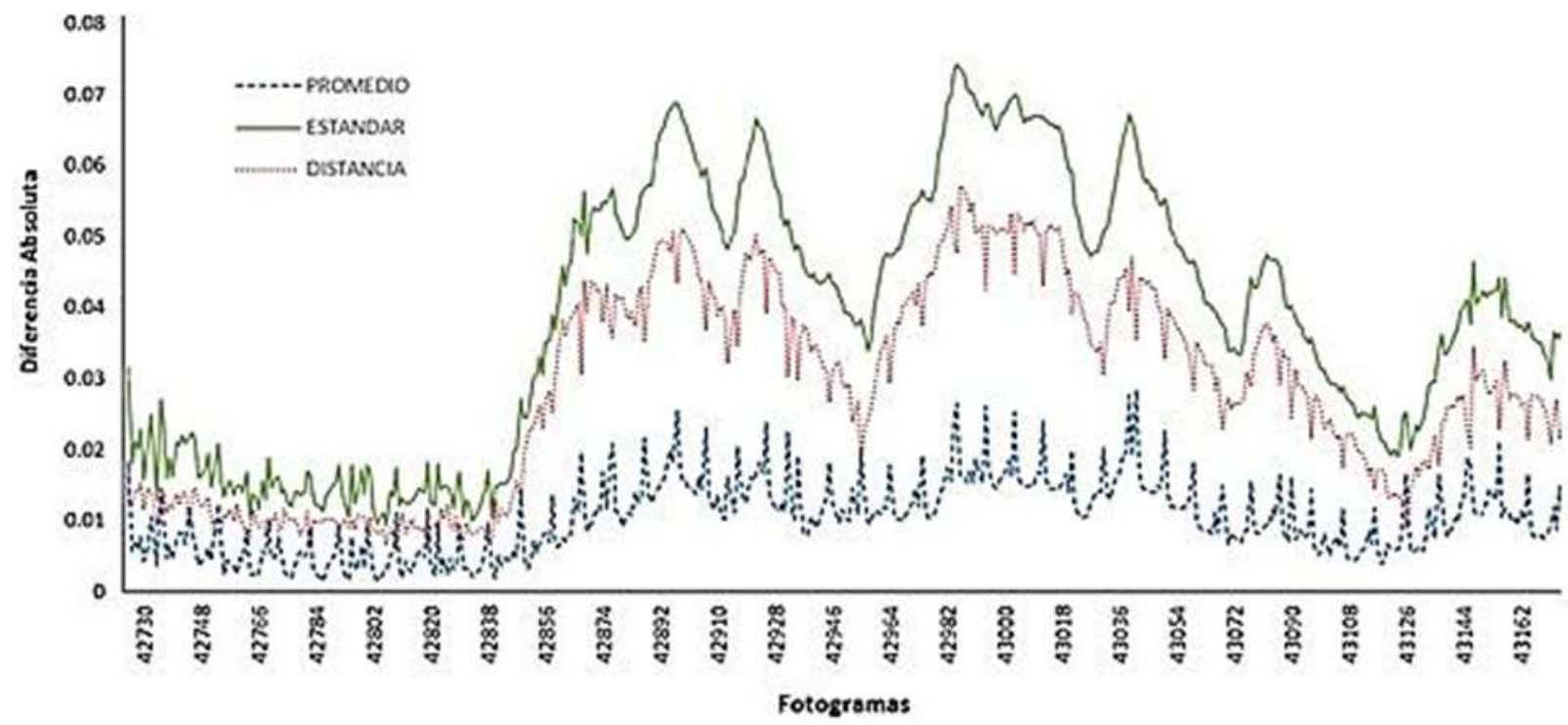

Fig. 3 Grafica del Valor promedio, desviación estándar y la distancia entre estos parámetros. 
En el eje $x$ de la Fig. 3 se muestra el tiempo o el número de fotogramas. Cuando el valor promedio y la desviación estándar son cercanos a cero, los fotogramas corresponden a escenarios donde no existen objetos en movimiento y las variaciones en estos fotogramas corresponden a ruido, por esta razón el valor de $k^{t}$ de la ecuación (3) tiene valores más elevados lo que permite discriminar entre objetos de interés y ruido. Sin embargo si la desviación se encuentra en valores superiores a 0.003 en la figura 3 , la secuencia de video en el tiempo t muestra escenarios con objetos en movimiento, por lo que el valor de $k^{t}$ debe ser pequeño. Experimentalmente se obtuvieron valores para $k^{t}$ desde 0.5 a 4.5 divididos en seis intervalos. Una vez que la imagen se ha modificado se aplica un filtro definido por

$M P_{i, j}(t)=M O_{i, j}(t)\left[\frac{1}{N^{2}} \sum_{x, y=-\left|\frac{N}{2}\right|}^{\left|\frac{N}{2}\right|} M O_{i+x, j+y}(t)\right]$

donde $\mathrm{N}$ es el tamaño de la ventana del filtro, el cual debe ser un número impar para que el pixel a evaluar sea el pixel central. Se puede notar que la función del filtro es parecida a la de un filtro promedio pesado por el pixel central. De la ecuación (6) se tiene que si el pixel central $M O_{i, j}^{t}$ es cero, permanecerá como cero y en caso de ser uno, se obtiene un valor promedio. Dependiendo del umbral usado $U 2^{t}$, es posible eliminar parte del ruido que aparece aleatoriamente

$$
\begin{aligned}
& M F_{i, j}(t)= \begin{cases}1, & M P_{i, j}(t) \geq U_{2} \\
0, & \text { Otro caso }\end{cases} \\
& U_{2}(t)=\frac{k_{2}(t)}{N^{2}}
\end{aligned}
$$

donde $k_{2}^{t}$ debe ser menor que $N^{2}$. Una vez que se ha eliminado el ruido se eliminan los objetos que tengan menos de 35 pixeles conectados

$$
\operatorname{Ccont}_{i, j}(t)= \begin{cases}1, & M F_{i, j}(t) \geq 35 \text { pixeles } \\ 0, & \text { otro caso }\end{cases}
$$

Dado que los pixeles que componen a un objeto de interés presentan colores similares, en particular en el caso de la detección de movimiento de un vehículo, algunos pixeles que componen el objeto son eliminados por los umbrales anteriores, además de que algunos objetos pueden ser divididos en dos o más partes. Por esta razón es necesario calcular los bordes de los objetos. Para extraer los bordes de los objetos de interés, se usa un algoritmo denominado "Canny" (Canny, 1986), para seleccionar los bordes en la región de interés se realiza una operación morfológica de dilatación sobre los pixeles considerados movimiento con un elemento estructural en forma de círculo con un diámetro correspondiente al $12 \%$ aproximadamente de la imagen original (diez pixeles), y se nombran como $C D_{i, j}^{t}$ de este modo se obtiene el borde del objeto de interés calculado en el fotograma en el tiempo t. Algunas regiones que contienen objetos de interés se dilataron y abarcan una región más amplia que el área de los objetos de interés, y por tanto se detectan bordes que no corresponden a dichos objetos. Sin embargo, dado que éstos bordes no se encuentran conectados a los objetos de interés y son relativamente pequeños, se eliminan aquellos bordes menores al $0.02 \%$ (15 pixeles) conectados, y así se eliminan parte de los bordes que no corresponden al objeto de interés, mientras que los bordes que superen este porcentaje se consideran parte del objeto de interes. Ahora se realiza una operación lógica "and" (\&), entre las componentes conectadas mayores al $0.04 \%$ (35 pixeles) y los fotogramas t y t-1 (10) y (11) la cual esta dada por:

$$
\begin{aligned}
& F C_{i, j}(t)=F_{i, j}(t) \& \operatorname{Ccon}_{i, j}(t) \\
& F C_{i, j}(t-1)=F_{i, j}(t-1) \& \operatorname{Ccon}_{i . j}(t)
\end{aligned}
$$

Efectuando una nueva diferencia absoluta sobre los fotogramas con la primera aproximación de movimiento por medio de los componentes conectados dadas por: 


$$
D F C_{i, j}(t)=\left|F C_{i, j}(t)-F C_{i, j}(t-1)\right|
$$

Considerando un nuevo umbral para discriminar movimiento significativo del ruido, es necesario calcular nuevamente los parámetros estadísticos de $D F C_{i, j}^{t}(12)$ a fin de obtener una nueva desviación estándar y valor promedio, para usarlos en el nuevo umbral dado por:

$$
\begin{aligned}
& M O C_{i, j}(t)= \begin{cases}1, & D F C_{i, j}(t) \geq U_{3}(t) \\
0, & \text { Otro caso }\end{cases} \\
& U_{3}(t)=\overline{C x(t)}+0.80 C \sigma(t)
\end{aligned}
$$

donde $\overline{C x(t)}$ y $C \sigma(t)$ son el valor medio y la varianza de $D F C_{i, j}(t)$, respectivamente. En la ecuación (14) se mantiene un valor 0.80 multiplicando a la desviación estándar, esto es porque las variaciones de la diferencia absoluta en las tramas, muestra un comportamiento más constante, dado a que se eliminó la mayor cantidad de ruido. Finalmente el objeto de interés es determinado mediante borde(t), que fue calculado con el algoritmo "Canny" y el movimiento calculado en (13). Finalmente se utiliza un umbral para eliminar objetos menores a un tamaño que se considere de un vehículo, en este caso consideramos que el objeto de interés, definido en (15), debe tener un mínimo de 200 pixeles.

$$
O I_{i, j}(t)=M O C_{i, j}(t) \& b o r d e(t)
$$

\section{RESULTADOS Y DISCUSIÓN}

Las figuras 4 y 5 presentan los resultados obtenidos en cada una de las etapas del método propuesto:

\section{Fotograma 1 (figura 4)}

a) Fotograma número 42277 b) Binarización de la región de movimiento usando (3); c) Eliminación de ruido usando (7) y (9); d) Operación morfológica de dilatación sobre la primera aproximación de movimiento; e) Detección de bordes en la región dilatada; f) Eliminación de bordes con un tamaño menor a 15 pixeles conectados; g) Diferencia absoluta realizada sobre la región dilata; h) Eliminación de ruido mediante (6); i) combinación de bordes y diferencia absoluta. j) Fotograma 42277, objetos de interés finales habiendo eliminando objetos menores a 200 pixeles conectados.

\section{Fotograma 2 (figura 5)}

a) Fotograma 42503; b) Binarización de la región de movimiento usando (3); c) Eliminación de ruido usando (7) y (9) ; d) Operación morfológica de dilatación sobre la primera aproximación de movimiento; e) Detección de bordes en la región dilatada; f) Eliminación de bordes con un tamaño menor a 15 pixeles conectados; g) Diferencia absoluta realizada sobre la región dilata; h) Eliminación de ruido mediante (6); i) combinación de bordes y diferencia absoluta, j) Fotograma 42503, objetos de interés finales, eliminando objetos menores a 200 pixeles conectados.

En las Figs. 4a) y 5a) se muestran dos fotogramas. En las Figs. 4b) y 5b) se observa la diferencia entre 2 fotogramas consecutivos, las Fig. 4c) y $5 c$ ) la detección del objeto de interés con una primera eliminación de ruido, pero se observa que la diferencia absoluta entre fotogramas (DAF) separa en regiones el objeto de interés, y es necesario complementar el método DAF con otro método (Kadiķis, \& Kārlis, 2013) para enfrentar el fenómeno de apertura en el primer plano (Utasi, \& Czúni, 2007). Al aplicar una dilatación morfológica en las regiones consideradas movimiento, se incluye el objeto de interés completo Figs. 4d) y 5d). Ahora al calcular el borde en las regiones ampliadas se obtienen los bordes de los objetos de interés. Dado que la región se encuentra ampliada sobre el objeto de interés, algunos bordes que no corresponden a los objetos de interés se detectan, Figs. 4e) y 5 e), con una eliminación de los bordes menores a 15 pixeles conectados, algunos bordes que no corresponden al objeto se eliminan como se muestra en las figuras $4 \mathrm{f}$ ) y $5 \mathrm{f}$ ). Al combinar la detección de bordes con la segunda DAF realizada en las regiones dilatas, figuras $4 \mathrm{~g}$ ), h) y $5 \mathrm{~g}$ ), h), las partes del objeto detectadas en la segunda DAF se unen en uno solo, Figs. 4i) y $5 \mathrm{i}$ ). Finalmente se utiliza un umbral para eliminar regiones con menos de 200 pixeles $(0.2 \%)$, y eliminar objetos que no corresponden a vehículos, e incluso, si algún objeto no se unió completamente, las partes restantes no unidas son pequeñas, y este umbral permite eliminarlas como se muestra en las Figs. 4j) y 5j). 


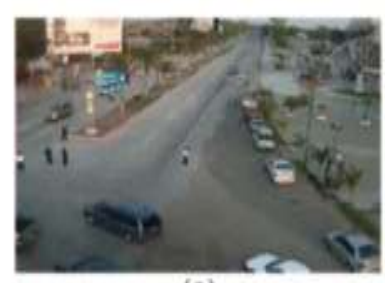

(a)
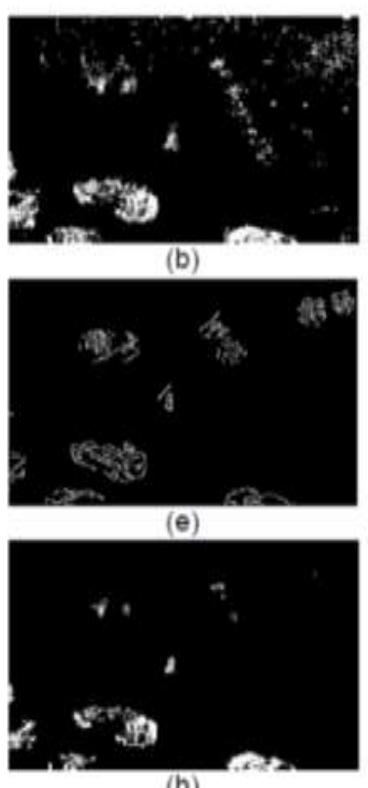

(h)
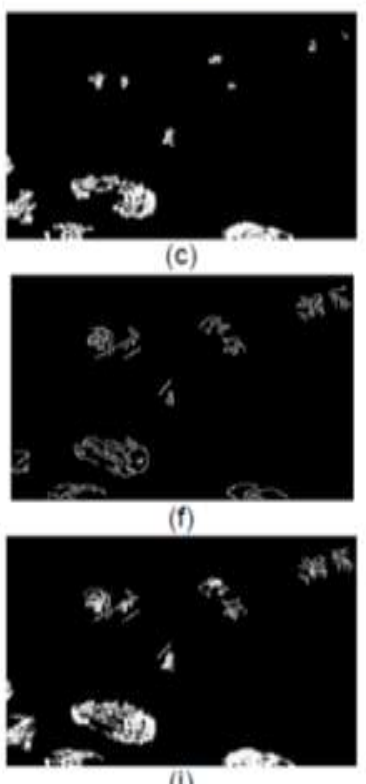
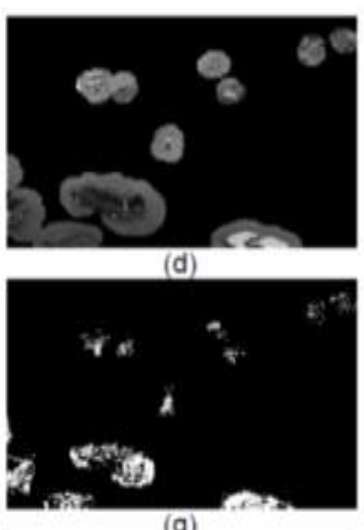

(9)

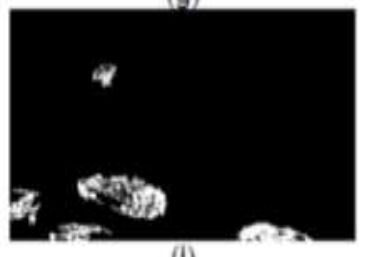

Fig. 4: Etapas del procesamiento de fotograma 1

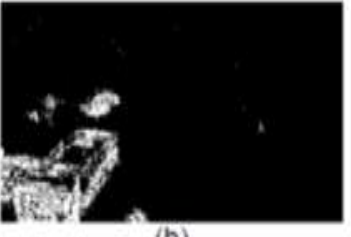

(b)

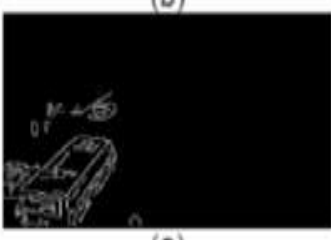

(e)

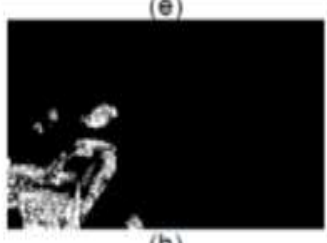

(h)

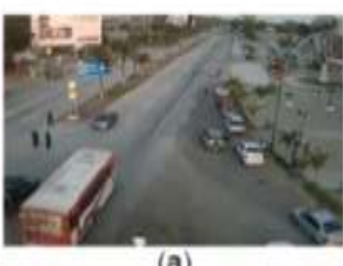

(a)

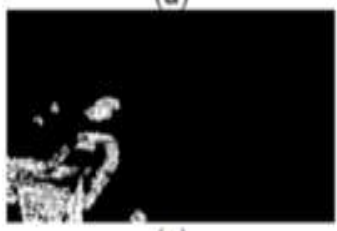

(c)

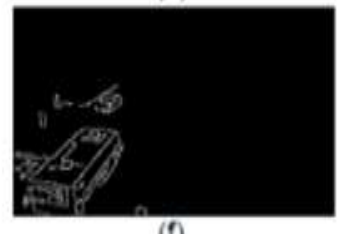

(f)

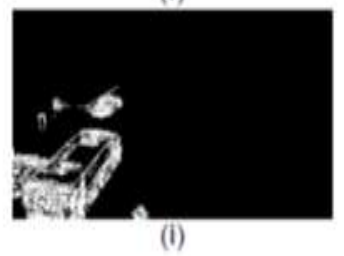

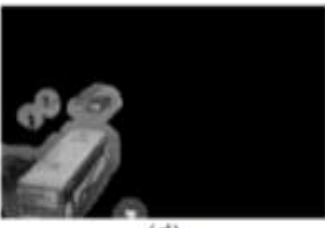

(d)

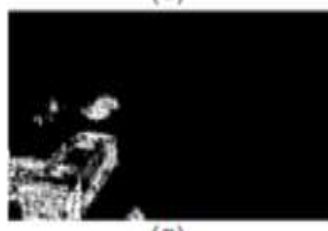

(g)

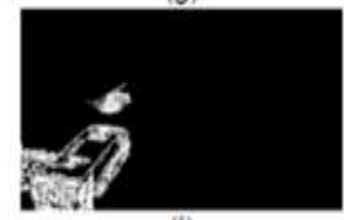

(j)

Fig. 5. Etapas del procesamiento de fotograma 2 


\section{CONCLUSIONES}

De los resultados obtenidos y su análisis, se concluye que: El método de diferenciación entre fotogramas requiere un procesamiento adicional que permita solucionar el problema de apertura, dado que el problema consiste en unir las partes del objeto detectado, el método presentado permite la unión de estos objetos usando la detección de bordes. Sin embargo debido a que la detección de bordes se realiza en un entorno más amplio que la detección, el contorno del objeto de interés puede verse modificado por los bordes detectados en las cercanías. Las siluetas de los objetos de interés obtenidas mediante este algoritmo, presentan las características morfológicas del objeto que representan, no están distorsionadas de forma que para un observador humano le sea difícil determinar de que tipo de vehículo se trata, así aprovechando esta característica, como trabajo a futuro, se considerara usar estos resultados para clasificar en grupos los vehículos, por ejemplo, pequeño, mediano, grande y tracto camiones de doble remolque.

\section{AGRADECIMIENTOS}

Esta investigación fue financiada por el Consejo Nacional de Ciencia y Tecnología (CONACyT) y por el Instituto Politécnico Nacional.

\section{REFERENCIAS}

Buch, N., Velastin S. y Orwell J., A review of computer vision techniques for the analysis of urban traffic, IEEE Transactions on Intelligent Transportation Systems, 12 (3), 920-939 (2011).

Canny, J., A Computational Approach to Edge Detection, Pattern Analysis and Machine Intelligence, IEEE Transactions Pattern Analysis and Machine Intelligence, 8 (6), 679,698, 1986

Cao, J. y Li L., Vehicle objects detection of video images based on gray-scale characteristics, Education Technology and Computer Science, ETCS'09, First International Workshop on Education Technology and Computer Science, 2, 936-940 (2009).

Kadiķis, R. y Kārlis F., Efficient video processing method for traffic monitoring combining motion detection and background subtraction, Proceedings of the Fourth International Conference on Signal and Image Processing, 131-141 (2012).

Mendes, V., Leta F., Conci A. y Gonçalves L., Detección de posición angular de embarcaciones, utilizando técnicas de visión Computacional y redes neurales artificiales, Revista Información Tecnológica, 21(6) 177188 (2010).

Padmadas, M., Krishnan N., Mualidharan V., y Ravikumar P., A deployable architecture of Intelligent Transportation System-A developing country perspective, IEEE International Conference on Computational Intelligence and Computing Research, 1-6. IEEE (2010).

Salinas-Salas G, Stintz M., Wessely B., Aplicación del método fotométrico para la detección de la distribución de tamaño de Micro partículas, Revista Información Tecnológica, 16(4), 59-68 (2005).

Utasi, Á., y L. Czúni, Reducing the foreground aperture problem in mixture of Gaussians based motion detection, 14th IEEE International Workshop on Systems, Signals and Image Processing, 157-160, (2007).

Wang, Guolin, Deyun Xiao, y Jason Gu, Review on vehicle detection based on video for traffic surveillance, IEEE International Conference on Automation and Logistics, 2961-2966 (2008).

Xiaofeng, L., Zhang T., y Liu Z., A novel method on moving-objects detection based on background subtraction and three frames differencing, International Conference on Measuring Technology and Mechatronics Automation, 1, 252-256 (2010).

Zhang, T., Zaiwen L., Xiaofeng L., y Xiaoyi W., Study on moving-objects detection technique in video surveillance system, Chinese Control and Decision Conference, 2375-2380 (2010). 